\title{
Vestibular effects on cerebral blood flow
}

\author{
Jorge M. Serrador ${ }^{1}$ \\ Todd T. Schlegel ${ }^{2}$ \\ F. Owen Black ${ }^{3}$ \\ Scott J. Wood ${ }^{2,4}$ \\ ${ }^{1}$ Harvard Medical School, Beth Israel Deaconess Medical Center, Boston, MA \\ ${ }^{2}$ NASA Johnson Space Center, Houston, TX \\ ${ }^{3}$ Neurotology Research, Legacy Health System, Portland, OR \\ ${ }^{4}$ Universities Space Research Association, Houston, TX
}

RUNNING HEAD: Vestibular effects on Cerebral Blood Flow

Word Count

Abstract: $\quad 287$

Narrative: 1799

Methods: $\quad 475$

References: 20

Tables: $\quad 0$

Figures: $\quad 4$ 
Humans demonstrate a number of unique adaptations that allow the maintenance of blood pressure and brain blood flow after transition to the upright position. While these adaptations maintain heart-level mean arterial pressure similar to supine values, the brain remains $\sim 30 \mathrm{~cm}$ above the heart, resulting in a $\sim 25 \%$ decrease in perfusion pressure. To maintain brain blood flow, the cerebral vessels must dilate in response to this change in position. While several physiological systems are involved in adaptation to the upright posture, including cerebral autoregulation, the unique role that the vestibular system plays in helping to maintain brain blood flow is just beginning to be elucidated. Since the vestibular system not only assists in balance control and locomotion but provides direct information about the body's position relative to gravity, it can, within milliseconds, detect a change in posture. Thus it is possible that a vestibular signal indicating upright could assist in this necessary cerebral vasodilation. In this work we demonstrate a direct effect of vestibular activation on cerebral blood flow regulation. By stimulating the otoliths, the organs that sense gravity, using sinusoidal translation or tilt in the dark at five frequencies, we found that cerebral blood flow was modulated according to the frequency of stimulation. In addition, changes in cerebral blood flow were in opposition to blood pressure changes, likely indicating a direct effect of otolith activation on cerebral blood flow regulation. We anticipate these findings may lead to new treatment modalities for cerebral hypoperfusion under a variety of circumstances. For example, with aging there is well documented vestibular loss that might contribute to a general age-associated reduction in global cerebral blood flow. Similarly, patients with orthostatic intolerance could have vestibular impairment that exacerbates cerebral hypoperfusion when upright. 
To maintain arterial pressure when upright humans must respond to a translocation of blood from the upper body into the lower limbs. Responses to this translocation include baroreflex-mediated increases in heart rate and in peripheral vasoconstriction, thus compensating for reduced venous return and minimizing pooling of blood in the lower body. Previous work in animals has demonstrated that sectioning the vestibular nerve to remove information on position relative to gravity results in a dramatic increase in postural hypotension. ${ }^{1}$

While a role for the vestibular system in the autonomic response to the upright position has been documented, there have been no studies demonstrating a direct effect of otolith stimulation on cerebral blood flow. Anatomical evidence demonstrates that neural connections are present between the vestibular nuclei and cerebral vessels through two possible pathways (Figure 1). Connection have been found between the Vestibular Nuclei and the Fastigial Nucleus, ${ }^{2}$ then to the Rostral Ventrolateral Medulla, ${ }^{3}$ followed by vasodilatory connections to the cerebral vessels. ${ }^{4}$ Similarly, neurons travel from the Vestibular Nuclei to the Nucleus Tractus Solitarius ${ }^{5}$ and then to the Pterygopalatine Ganglion, ${ }^{6}$ resulting in cerebral vasodilation. ${ }^{7}$ However, the role these connections play with respect to adjustments to posture remains to be determined.

Caloric vestibular stimulation in humans that activates the semicircular canals, involved with detection of movement (i.e. angular acceleration), has been found to increase blood flow in the basilar ${ }^{8}$ and middle cerebral arteries ${ }^{9}$ as well as the parietal lobe $^{10}$ while decreasing flow in the posterior cerebral artery. ${ }^{9}$ However, it remains unclear whether these changes were due to functional activation of vestibular and other centers, rather than general vascular changes.

Our group recently found that subjects exposed to 30 minutes of hypergravity demonstrate impaired cerebral blood flow regulation that returned to normal upon 
assumption of the upright posture. ${ }^{11}$ Furthermore, this impairment was found to correlate with non-invasive measures of otolith sensitivity, providing indirect evidence of a role for otolith activation. Similarly, using head down neck flexion, we found a modulation of cerebrovascular resistance ${ }^{12}$ that may have been due to otolith activation. Finally, in subjects that developed nausea during centrifugation, cerebral blood flow was reduced almost two minutes prior to actual nausea. ${ }^{13}$ Since centrifugation was performed in the dark with no visual cues, these data suggest a role for vestibular inputs in affecting the cerebrovasculature.

In search of more direct evidence for an otolith role in cerebral blood flow regulation, we investigated the responses of 24 subjects (11 males, 13 females, age $29 \pm 9$ years, $173 \pm 9 \mathrm{~cm}, 72 \pm 12 \mathrm{~kg}$ ) to translational motion during centrifugation or pitch tilt at five frequencies $(0.5,0.25,0.125,0.0625$ \& $0.03125 \mathrm{~Hz})$. During centrifugation (Figure 2B) subjects were rotated at a constant rate (250 deg/sec) for 5 min to allow the horizontal canal response to decay, and then translated back and forth at each frequency so that the resultant gravitoinertial force vector (combined translation and centrifugal) tilted \pm 25 degree with respect to the subject. During the tilt protocol (Figure 2A) subjects were tilted \pm 25 degree at each frequency about an interaural axis. All protocols were performed in the dark in the pitch plane so subjects perceived a sensation of tilt and/or translation back and forth. It's important to note that the variable radius centrifugation elicited otolith activation without stimulating the canals, while tilts about an Earth-horizontal axis elicited both otolith and canal cues.

Both centrifugation and tilt resulted in changes in cerebral blood flow velocity (CBFV) as measured by transcranial Doppler that were linked to stimulation frequency (Figure 3). CBFV responses were dependent on frequency of stimulation $(\mathrm{P}<0.01)$ and demonstrated significant changes within each frequency cycle $(\mathrm{P}<0.01)$ that differed by frequency (Frequency x Cycle interaction, $\mathrm{P}<0.01$ ). While CBFV during centrifugation 
at $0.5 \mathrm{~Hz}$ tended to be lower than during tilt $(\mathrm{P}=0.07)$, the change within the cycle was very similar. The CBFV responses shown in Figure 3 may have been due to factors unrelated to the otoliths, such as driving pressure or changes in arterial $\mathrm{CO}_{2}$ levels. For example, there was a significant effect on blood pressure at brain level of both frequency of stimulation $(\mathrm{P}<0.01)$ and position within cycle $(\mathrm{P}<0.01)$ that differed by frequency (Frequency x Cycle interaction, $\mathrm{P}<0.01$ ). In addition, blood pressure was significantly higher during centrifugation as compared to tilt at all frequencies $(\mathrm{P}<0.01)$. However, the pattern of the blood pressure and CBFV changes were different. For example, as shown in the left panel $(0.03125 \mathrm{~Hz})$ of Figure 3, CBFV increased during both the $+25^{\circ}$ and $-25^{\circ}$ tilt positions whereas blood pressure increased only during the $+25^{\circ}$ tilt position. Similarly at 0.25 and $0.5 \mathrm{~Hz}$, blood pressure increased during the first half of the cycle (i.e. moving from upright to pitch forward and back to upright) at the same time CBFV decreased. Thus CBFV was decreasing even though driving pressure was increasing. Thus, while blood pressure consistently increased somewhere between a quarter (slowest frequency) and half way into the cycle (fastest frequency), CBFV demonstrated bimodal peaks at the slowest frequency and decreases at the fastest frequency (Figure 3). This would again suggest a disparity in the response of the two variables. These data therefore suggest that otolith activation at various frequencies likely directly affects cerebral blood flow.

Examining end tidal $\mathrm{CO}_{2}$ changes, an indicator of arterial $\mathrm{CO}_{2}$, demonstrates similar disconjugate patterns. End tidal $\mathrm{CO}_{2}$ was also affected by both frequency of stimulation $(\mathrm{P}<0.01)$ and position within cycle $(\mathrm{P}<0.01)$ that differed by frequency (Frequency x Cycle interaction, $\mathrm{P}<0.01$ ). In addition end tidal $\mathrm{CO}_{2}$ was significantly lower during centrifugation with translation at $0.5 \mathrm{~Hz}$. Examination of individual frequencies showed differing patterns. For example, at $0.03125 \mathrm{~Hz}$, end tidal $\mathrm{CO}_{2}$ increased at $+25^{\circ}$ and decreased at $-25^{\circ}$, while cerebral blood flow increased at both $+25^{\circ}$ and $-25^{\circ}$. Similarly at 0.25 and $0.5 \mathrm{~Hz}$, end tidal $\mathrm{CO}_{2}$ did not change within the 
cycle, while cerebral blood flow decreased significantly. These data demonstrate that changes in end tidal $\mathrm{CO}_{2}$ cannot completely explain position-related changes in cerebral blood flow.

While there was no significant difference in responses to either sinusoidal tilt or translation during centrifugation at the four slowest frequencies, CBFV was $6.8 \pm 0.1 \%$ lower during centrifugation. Since end tidal $\mathrm{CO}_{2}$ was also $1.6 \pm 0.1 \mathrm{mmHg}$ lower, based on the cerebrovascular reactivity of $2.6 \% / \mathrm{mmHg}$, approximately two thirds of the $6.8 \%$ decrease could be explained by the centrifugation related hypocapnia.

Since changes in CBFV during vestibular stimulation are likely mediated through changes in CVR. Since cerebral blood flow is normally regulated to maintain flow relatively constant in the face of changing perfusion pressure-a phenomenon known as cerebral autoregulation ${ }^{14}$ — changes in CVR could be both the result of changes in blood pressure (autoregulation) as well as changes in chair position (vestibular). Figure 3 demonstrates that changes in CVR within the motion cycle were similar to the changes in blood pressure, suggesting an autoregulatory response. However, if CVR changes were solely autoregulatory in nature, CBFV would have remained constant throughout motion. The fact that cerebral flow velocity was changing throughout the cycles indicates CVR changes were not sufficient to maintain flow indicating that a nonautoregulatory component was influencing CVR and causing changes in flow.

We believe that these data are the first to show that responses in CBFV during centrifugation and tilt stimuli cannot be fully accounted for by change in either mean arterial blood pressure and/or end tidal $\mathrm{CO}_{2}$. Since similar findings were obtained during both centrifugation involving otolith cues and tilt stimuli involving otolith and canal cues, the changes in CBFV are therefore likely dependent on otolith stimulation in particular. 
Consistent with previous studies, ${ }^{15}$ the perception of tilt decreased as a function of stimulus frequency, with tilt perception being very similar between centrifugation and tilt stimuli at lower frequencies when canal inputs were negligible due to reduced roll velocity. Subjects reported $52 \pm 7^{\circ}$ of perceived tilt during both tilt and translation at $0.03125 \mathrm{~Hz} ; 42 \pm 6^{\circ}$ during tilt and $37 \pm 6^{\circ}$ during translation at $0.0625 \mathrm{~Hz} ; 33 \pm 5^{\circ}$ during tilt and $27 \pm 5^{\circ}$ during translation at $0.125 \mathrm{~Hz} ; 22 \pm 4^{\circ}$ during tilt and $19 \pm 4^{\circ}$ during translation at $0.25 \mathrm{~Hz}$; and $18 \pm 3^{\circ}$ during tilt and $9 \pm 3^{\circ}$ during translation at $0.5 \mathrm{~Hz}$.

\section{Discussion}

Our overall results demonstrate a frequency-dependent response to otolith stimulation in cerebral blood flow that is in part independent of changes in both mean arterial pressure and end tidal $\mathrm{CO}_{2}$.

Interestingly, vestibular activation had two main effects. First, changes in cerebral blood flow velocity in the low frequency range $(<0.25 \mathrm{~Hz})$ were especially correlated to position (Figure 4, left panel), whereas those in the high frequency range $(>=0.25 \mathrm{~Hz}$ ) were especially correlated to the velocity of motion (Figure 4, right panel). Correlations between cerebral blood flow velocity and the velocity of motion were significantly lower for the lower frequency ranges compared to the higher frequency ranges for both tilt and centrifugation $(<0.4$ and $\sim 0.6$, respectively). A generally opposite pattern was demonstrated for position (left panel), where correlations between cerebral blood flow velocity and position were $\sim 0.6$ for tilt at $0.0625 \& 0.125 \mathrm{~Hz}$, decreasing to $\sim 0.4$ at the higher frequencies. Interestingly, at the lowest frequency for position, the correlation was only 0.2. One possible explanation is that at the lowest frequency the response depended on absolute tilt angle (i.e. tilt from vertical in either direction), rather than 
relative (i.e. forward tilt vs. backward tilt). However, correlating cerebral flow velocity to absolute tilt only increased the $\mathrm{R}^{2}$ to $0.25 \pm 0.5$. Furthermore, during centrifugation the correlations did not change significantly across frequencies. The changing correlations during tilt may reflect the influence of vertical canals indirectly through canal-otolith integration at the site of the vestibular nuclei. An alternate explanation may be that during tilt at very low frequencies (i.e. $0.03125 \mathrm{~Hz}$ ), other somatosensory cues also influence the cerebral blood flow response, thus masking vestibular effects whereas the novel experience of centrifugation produced similar correlations across all frequencies

The second major effect of vestibular stimulation was the direction-dependent effect at frequencies greater than $0.03125 \mathrm{~Hz}$. Movement from pitch forward (i.e. semiprone) to pitch backward (semi-supine) resulted in increases in cerebrovascular resistance and decreases in cerebral flow velocity (Figure 3). It remains unclear why there was a direction-dependent effect of pitch. However Cheung et al ${ }^{16}$ reported greater postural hypotension during roll vs. pitch tilts, suggesting the possibility that cardiovascular responses to tilt may be direction dependent. Similarly we previously found that cerebral blood flow changes during head position manipulation differed in the prone vs. supine position. ${ }^{12}$ Since the plane of the otolith maculae is tilted back about 20 deg relative to our upright position (Reid's baseline parallel to Earth-horizontal plane), the pitch forward tilts would bring the otoliths in a position of increased sensitivity whereas the pitch backward would bring them in a position of reduced sensitivity. $^{17}$

Regardless of the exact etiology of these responses, our data demonstrate the importance of vestibular inputs on the cerebral blood flow response to changes in position relative to gravity. 


\section{METHODS}

Subjects

Twenty four healthy, non-smoking subjects (29 \pm 9 years, $72 \pm 12 \mathrm{~kg}, 173 \pm 9 \mathrm{~cm}, 11$ females, 13 males) were recruited. The study protocol was approved by the Legacy Health Systems Institutional Review Board.

Experimental Protocol

Instrumentation: Beat-by-beat blood pressure was measured by photoplethysmographic cuff on a finger (Finapres, Ohmeda, $\mathrm{CO}$, USA). End-tidal $\mathrm{CO}_{2}$ was sampled from expired air via a nasal catheter (Puritan-Bennett, Wilmington, MA, USA). Cerebral flow velocity in the middle cerebral artery was obtained by transcranial Doppler (MultiDop T, DWL, Germany) as previously described. ${ }^{18}$ All physiologic signals were digitized at $500 \mathrm{~Hz}$ (Windaq, Dataq Instruments, OH, USA). Cerebrovascular reactivity was assessed during 3 min of resting ventilation, inspiration of $8 \% \mathrm{CO}_{2}, 21 \% \mathrm{O}_{2}$, balance nitrogen for $2 \mathrm{~min}$ and mild hyperventilation for $2 \mathrm{~min}$.

Tilt Protocol (Figure 2A): Subjects were seated upright in a high-torque hydraulic-powered tilt chair capable of delivering controlled angular accelerations of > $1,000 \% \mathrm{~s}^{2}$. Subject position was adjusted to align the interaural axis near the rotation axis such that the linear accelerations (both tangential and centripetal accelerations) at the otolith organs fell below the human acceleration threshold. ${ }^{19}$ The vestibular stimulation consisted of sinusoidal tilt $+/$ - 20 degrees in the pitch plane at five frequencies: $0.5 \mathrm{~Hz}$ (40 cycles); $0.25 \mathrm{~Hz}$ (20 cycles); $0.125 \mathrm{~Hz}$ (10 cycles); $0.0625 \mathrm{~Hz}$ (5 cycles) and $0.03125 \mathrm{~Hz}$ (5 cycles) with 80 seconds rest in-between stimulation frequencies. 
Centrifugation Protocol (Figure 2B): Subjects were seated upright over the center of rotation on a short-arm centrifuge device driven by direct-drive motor ( $80 \mathrm{ft} \mathrm{lb}$ ). Subjects were restrained so as to minimize motion of their torso, legs and head during centrifugation and all centrifugation was performed in the dark to eliminate visual cues of orientation relative to gravity.

The protocol consisted of:

- 3 min baseline

- Acceleration at $25 \% \mathrm{~s}^{2}$ to $250^{\circ} / \mathrm{sec}$ over center of rotation for $5 \mathrm{~min}$

- Translation of the chair back and forth in the pitch plane at 5 frequencies:

$0.5 \mathrm{~Hz}(+/-6.2 \mathrm{~cm}) ; 0.25 \mathrm{~Hz}(+/-8.4 \mathrm{~cm}) ; 0.125 \mathrm{~Hz}$ (+/- $9.2 \mathrm{~cm}) ; 0.0625$

$\mathrm{Hz}$ and $0.03125 \mathrm{~Hz}(+/-9.4 \mathrm{~cm})$ using the same cycles and rest as tilt.

- Deceleration to complete stop at $25^{\circ} / \mathrm{s}^{2}$

- Six subjects that became nauseated were not included in the analysis.

Data processing and Analysis

Post-processing was done using custom-written MATLAB scripts (The Mathworks, Natick, MA). Cerebrovascular resistance was calculated from brain level blood pressure (derived using hydrostatic correction) and cerebral flow velocity.

Cerebrovascular reactivity was determined by a linear fit of beat-by-beat cerebral flow velocity with associated end-tidal $\mathrm{CO}_{2}$ values after incorporating the known 6 sec time delay between end-tidal CO2 changes and associated cerebral flow velocity response. ${ }^{20}$ The effects of stimulus (Tilt vs. Centrifugation) and Frequency on cerebral flow velocity, arterial pressure, end tidal $\mathrm{CO}_{2}$, and cerebrovascular resistance were assessed 
using a repeated-measures General Linear Model (SPSS, Chicago, IL, USA). Data are presented as mean \pm SEM. 
FIGURE LEGENDS

Figure 1 - Anatomical connections demonstrating possible pathways connecting vestibular organs and the cerebral vessels.

Figure 2-A) Diagrammatic representation of the relative chair position over the course of each cycle. B) Representation of movement into the pitch-forward position during centrifugation. Subjects were oscillated back at forth at 5 frequencies to produce sufficient centripetal acceleration to create a corresponding gravitoinertial acceleration (GIA) that produced similar perceptions of tilt to the pitch-tilt chair protocol.

Figure 3- Response of subjects to five frequencies of stimulation averaged over 40 cycles for $0.5 \mathrm{~Hz}, 20$ cycles for $0.25 \mathrm{~Hz}, 10$ cycles of $0.125 \mathrm{~Hz}$, and 5 cycles of $0.0625 \mathrm{~Hz}$ and $0.03125 \mathrm{~Hz}$. Cerebral flow velocity (CFV) in the middle cerebral artery was affected at all frequencies during sinusoidal translation in the pitch plane (providing a pitch tilt stimulus) while being rotated at 250 deg/sec (CEN - Centrifugation) as well as sinusoidal \pm 25 degree pitch tilt (TILT). Base represents mean of 3 min baseline while sitting quietly in both conditions and Rot represents three minute average while rotating on center during centrifugation.

Figure 4- Correlation between position (left panel) or velocity (right panel) and cerebral flow velocity (CFV) at the various frequencies. Changes in CFV were correlated to velocity only at the highest two frequencies for both tilt (TILT) and centrifugation (CEN). In contrast, changes in CFV were correlated to position at all frequencies for centrifugation. During tilt, changes were strongly correlated at $0.0625 \& 0.125 \mathrm{~Hz}$, with lower values at the higher and lowest frequencies. *, 
indicates significant difference from $0.5 \mathrm{~Hz}(\mathrm{P}<0.05) ; \alpha$, indicates significant difference from velocity correlation at that frequency $(P<0.05)$. 


\section{ACKNOWLEDGEMENTS}

The authors would like to thank Valerie Stallings and Julie Leduc for their assistance in data collection and analysis and Sarah La Rose in the creation of the illustration of anatomical connections.

\section{GRANTS}

This work was supported by NIDCD grants R03DC5545 (Serrador), R03DC5547 (Wood), R01DC0205 (Black) and a NASA grant NNJ04HI13G (Serrador).

\section{AUTHOR CONTRIBUTIONS}

J.S. and S.W. conceived the experiment, and together with F.O.B. carried it out; J.S designed and carried out the data analysis; J.S. and S.W. co-wrote the paper with scientific interpretation input from T.S. and F.O.B.

\section{AUTHOR INFORMATION}

Reprints and permissions information is available at npg.nature.com/reprintsandpermissions.

Correspondence and requests for materials should be addressed to serrador@hms.harvard.edu. 


\section{REFERENCES}

$1 \quad$ B. J. Yates and A. D. Miller, J Vestib Res 8 (1), 17 (1998).

2 A. G. Shaikh, F. F. Ghasia, J. D. Dickman et al., Journal of neurophysiology 93 (2), 853 (2005).

3 B. J. Yates, T. Goto, and P. S. Bolton, Brain Res 601 (1-2), 255 (1993).

$4 \quad$ E. V. Golanov, J. R. Christensen, and D. J. Reis, Neuroscience letters 288 (3), $183(2000)$.

5 B. J. Yates, L. Grelot, I. A. Kerman et al., The American journal of physiology 267 (4 Pt 2), R974 (1994).

$6 \quad$ K. Agassandian, V. P. Fazan, V. Adanina et al., The Journal of comparative neurology 452 (3), 242 (2002).

7 W. T. Talman, J. Corr, D. Nitschke Dragon et al., Auton Neurosci 133 (2), 153 (2007).

8 J.G. Heckmann, S. Leis, M. Muck-Weymann et al., Acta neurologica Scandinavica 100 (1), 12 (1999).

$9 \quad$ F.P. Tiecks, J. Planck, R.L. Haberl et al., J Cereb Blood Flow Metab 16 (6), 1379 (1996).

10 N. Takeda, K. Hashikawa, H. Moriwaki et al., J Vestib Res 6 (2), 127 (1996).

11 J. M. Serrador, S. J. Wood, P. A. Picot et al., J Appl Physiol 91 (5), 1986 (2001).

12 T. D. Wilson, J. M. Serrador, and J. K. Shoemaker, Brain Res 961 (2), 261 (2003).

13 J.M. Serrador, T. T. Schlegel, F. O. Black et al., Aviation, space, and environmental medicine 76 (2), 91 (2005). 
O. B. Paulson, S. Strandgaard, and L. Edvinsson, Cerebrovasc Brain Metab Rev 2 (2), 161 (1990).

15 S. J. Wood, M. F. Reschke, L. A. Sarmiento et al., Experimental brain research. Experimentelle Hirnforschung 182 (3), 365 (2007).

16 B. Cheung, K. Hofer, and L. Goodman, Aviation, space, and environmental medicine 70 (10), 966 (1999).

17 C. Fernandez and J. M. Goldberg, Journal of neurophysiology 39 (5), 970 (1976).

18 J. M. Serrador, P. A. Picot, B. K. Rutt et al., Stroke 31 (7), 1672 (2000).

19 G. M. Jones and L. R. Young, Acta Otolaryngol 85 (1-2), 45 (1978); A. J.

Benson, M. B. Spencer, and J. R. Stott, Aviation, space, and environmental medicine 57 (11), 1088 (1986).

20 M. J. Poulin, P. J. Liang, and P. A. Robbins, J Appl Physiol 81 (3), 1084 (1996). 


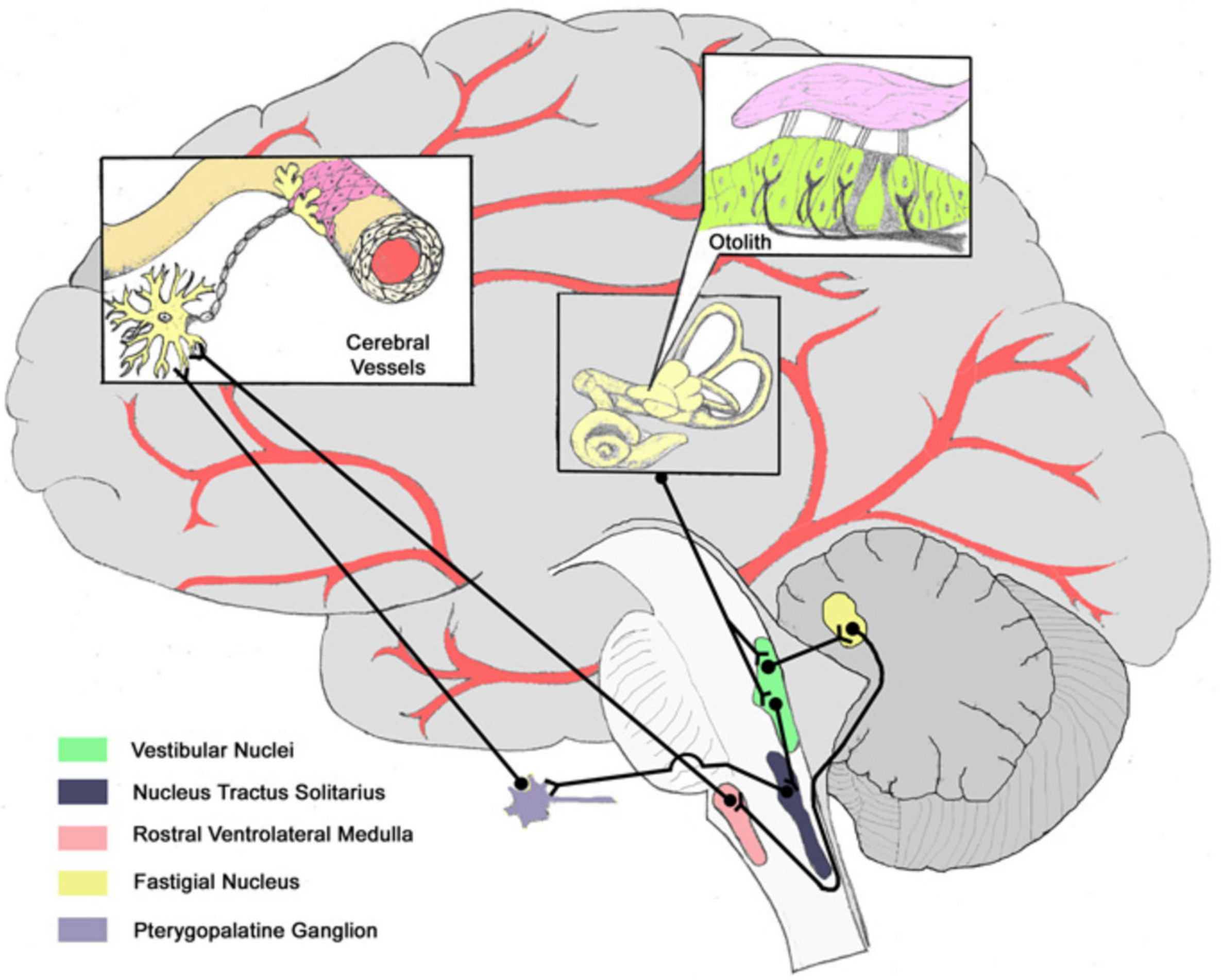


A

Pitch Forward

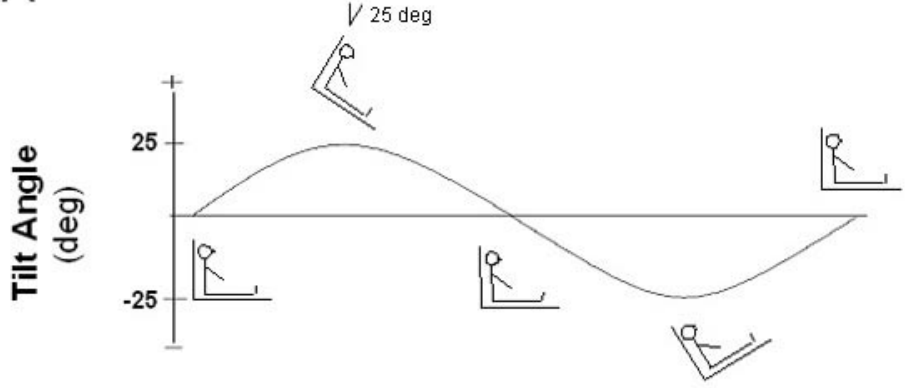

Pitch Backward

B

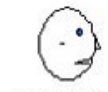

Subject Feels Upright
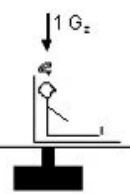
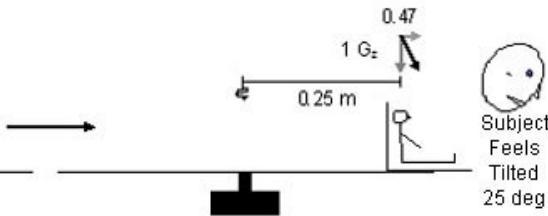

Subject Feels Tilted $25 \mathrm{deg}$ 


\section{Frequency of Motion $(\mathrm{Hz})$}

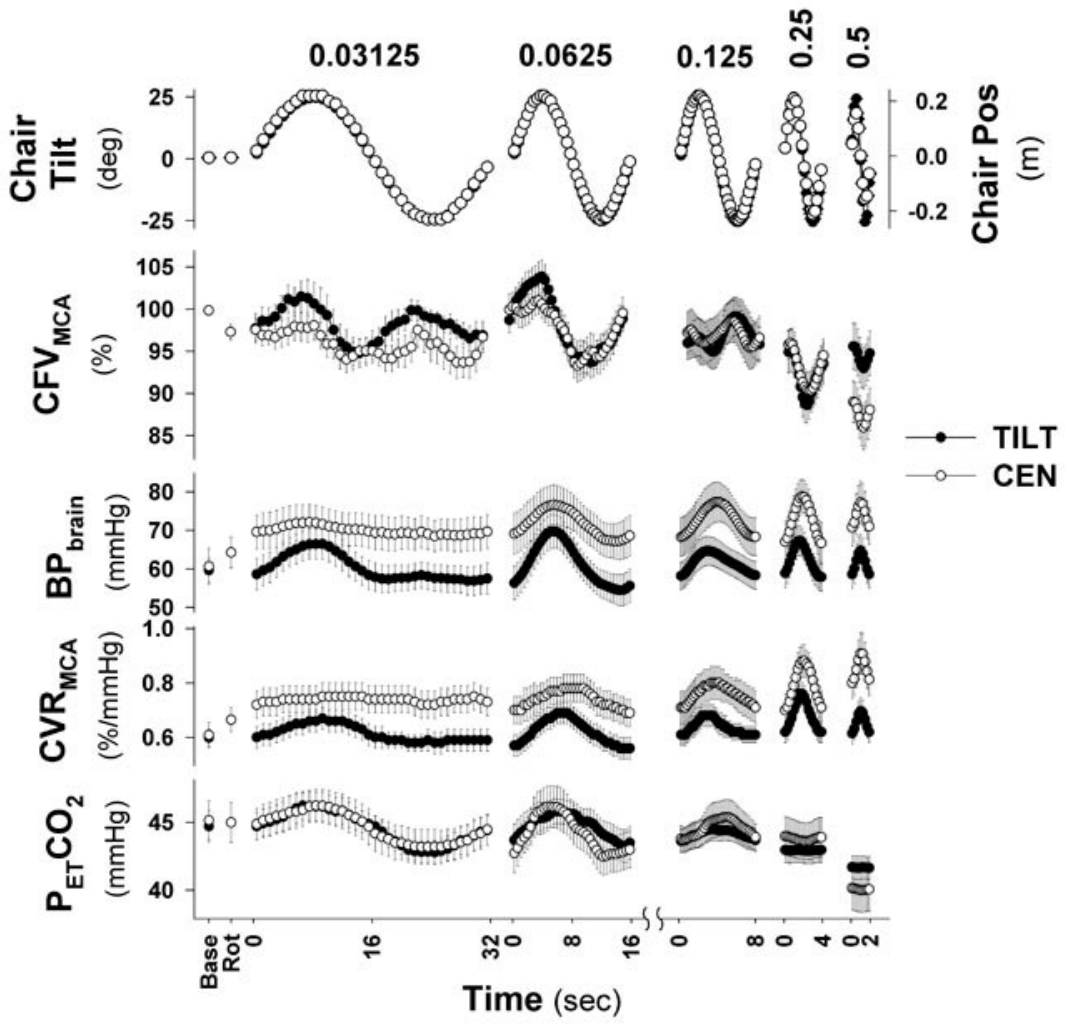




\section{Position}

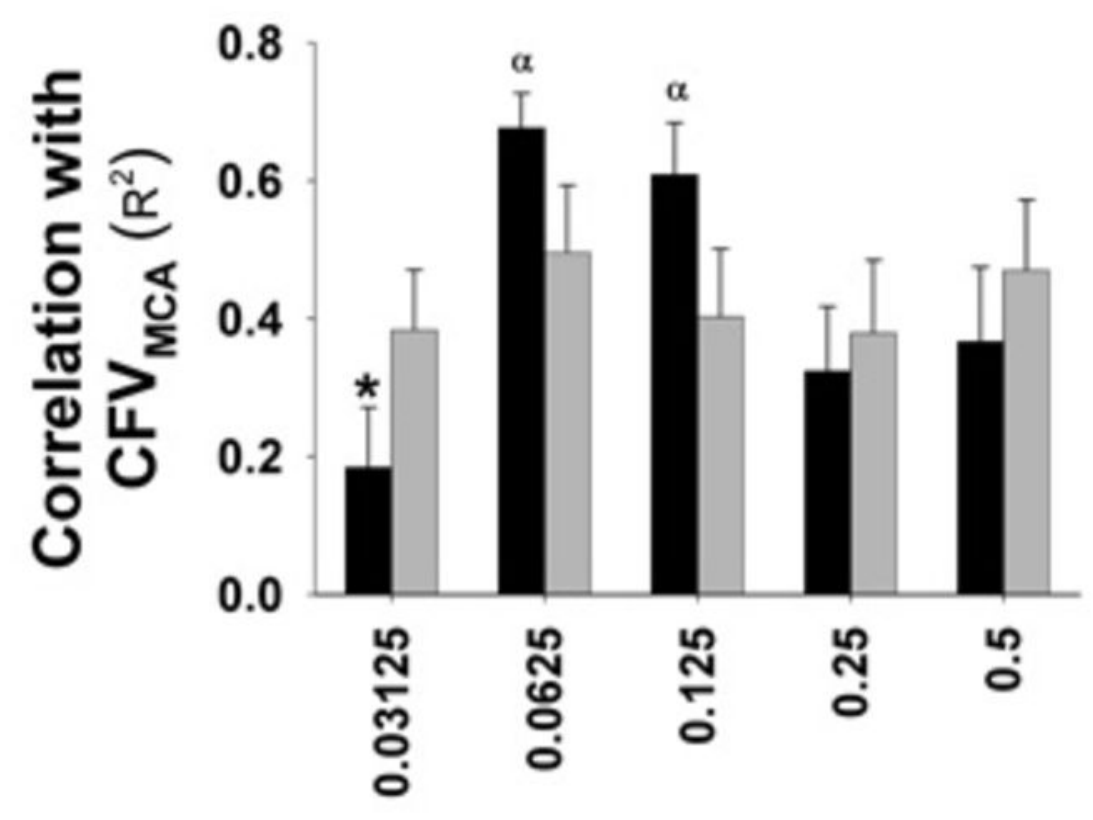

Velocity

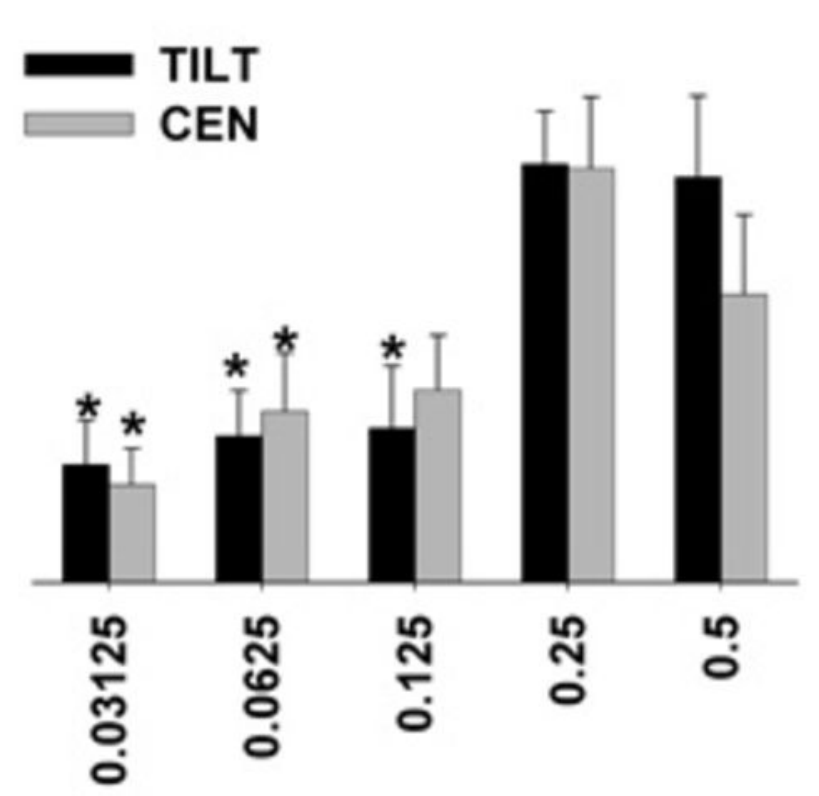

Frequency of Motion (Hz) 